\title{
Lepton flavour and lepton number violation searches at the LHCb experiment
}

\author{
Luca Pescatore* on behalf of the LHCb collaboration \\ École Polytechnique Fédérale de Lausanne \\ E-mail: luca.pescatore@cern.ch
}

\begin{abstract}
Recent hints for lepton-universality violation in $b \rightarrow c \ell v$ and $b \rightarrow s \ell \ell$ transitions could imply the existence of lepton-flavour violating $b$ decays. The LHCb experiment is well suited for the search for these decays due to its large acceptance and trigger efficiency, as well as its excellent invariant mass resolution and particle identification capabilities. Recent results on searches for lepton-flavour violating decays from the LHCb experiment are presented.
\end{abstract}

XXXIX International Conference on High Energy Physics

3-11 July 2018

Seoul, South Korea

${ }^{*}$ Speaker. 
The conservation of lepton flavour is well established by the non-observation of decays such as $\mu \rightarrow e \gamma$. However, it is not supported by strong theoretical reasons and it is already violated by the observation of neutrino oscillations. At the LHCb experiment, several searches for Lepton Flavour Violating (LFV) decays are performed including $B$-meson decays, but also $\Lambda_{b}$, charmed hadrons and lepton decays. These measurements are particularly interesting because anomalies in Lepton Flavour Universality (LFU) tests of the Standard Model (SM) were observed [1, 2, 3]. In several models, such as those involving lepto-quarks, LFU can be linked to LFV [4] and the branching ratios of flavour violating $b$ decays are predicted in the range $10^{-8}-10^{-10}$.

\section{1. $D^{0} \rightarrow e^{ \pm} \mu^{\mp}$}

The $D^{0} \rightarrow e^{ \pm} \mu^{\mp}$ decay is predicted in extensions of the $\mathrm{SM}$, for example in R-parity violating SUSY models, with a branching ratio that could be as high of $10^{-6}$ [5]. At LHCb this decay is searched using the full dataset from Run I, corresponding to $3 \mathrm{fb}^{-1}$ of integrated luminosity [3]. In order to reduce the combinatorics $D^{0}$ are tagged via the $D^{*} \rightarrow D^{0} \pi$ decay. The main challenge of this analysis is to distinguish signal and background from $D^{0} \rightarrow \pi^{ \pm} \pi^{\mp}$ decays. Most of such decays are rejected using particle identification requirements but an irreducible component, mainly due to the pions decaying in flight into muons, remains. As a further handle agains this background the fit to extract the yield is performed in two dimensions: the invariant mass of the $e \mu$ system, $m(e \mu)$, and the difference between the $D^{*}$ and $D^{0}$ masses. The result of the fit is shown in Fig. 1 . No significant signal excess is observed and a limit is set on the branching ratio using the CLs method at $\mathcal{B}\left(D^{0} \rightarrow e^{ \pm} \mu^{\mp}\right)<1.3 \times 10^{-8}$, at $90 \%$ confidence level, using the abundant $D^{0} \rightarrow K^{ \pm} \pi^{\mp}$ decay for normalisation. This is the best limit to date on this decay.
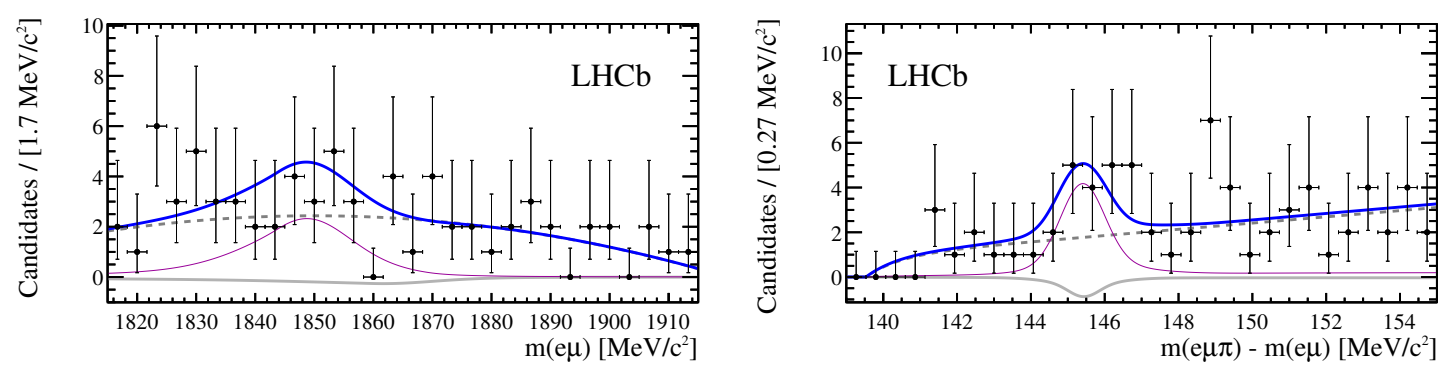

Figure 1: Invariant mass distribution of the reconstructed $D^{0} \rightarrow e^{ \pm} \mu^{\mp}$ candidates (right). The difference between the reconstructed $D^{*}$ and $D^{0}$ masses (left).

2. $B_{(s)}^{0} \rightarrow e^{ \pm} \mu^{\mp}$

The $B_{s}^{0} \rightarrow e^{ \pm} \mu^{\mp}$ and $B^{0} \rightarrow e^{ \pm} \mu^{\mp}$ decays are also searched at LHCb using $3 \mathrm{fb}^{-1}$ of data collected in Run I [6]. This analysis includes a special treatment of bremsstrahlung radiation emitted by the electron in the decay. Due to the bending of charged tracks, photons emitted before the calorimeter are absorbed by a different cell than the electron affecting the measurement of its momentum. A photon-recovery algorithm looks for clusters in the calorimeter which can be compatible with originating from a nearby electron track. The signal candidate invariant mass distribution 
shapes depend on the number of recovered photons. Furthermore, the background levels also differ, in fact, if a particle emitted photons, it is likely to be a real electron. For this reasons the analysis is done separately on two bremsstrahlung categories which are fitted simultaneously. The BDT is trained on simulated signal candidates and constructed such that its response for the signal decays is uniformly distributed between 0 and 1 . The realistic BDT response to the signal is measured on data using $B^{0} \rightarrow K^{ \pm} \pi^{\mp}$ candidates as a signal proxy as shown in Fig. 2. No cut is applied on the classifier output but the analysis is done in bins of it, which are fitted simultaneously. This method allows to retain a better sensitivity and furthermore it reduces the systematic uncertainty due to the estimation of the efficiency as this is calibrated on data rather then obtained from simulation. Figure 2 shows the fitted invariant mass distribution of the $e \mu$ system integrated over all considered categories. No excess is observed and limits are set at $\mathcal{B}\left(B_{s}^{0} \rightarrow e^{ \pm} \mu^{\mp}\right)<5.4(6.3) \times 10^{-9}$ and $\mathcal{B}\left(B^{0} \rightarrow e^{ \pm} \mu^{\mp}\right)<1.0(1.3) \times 10^{-9}$ at $90 \%$ (95\%) confidence level. These represent the best limits to date on these channels and the first limit for the $B_{s}^{0}$ channel.
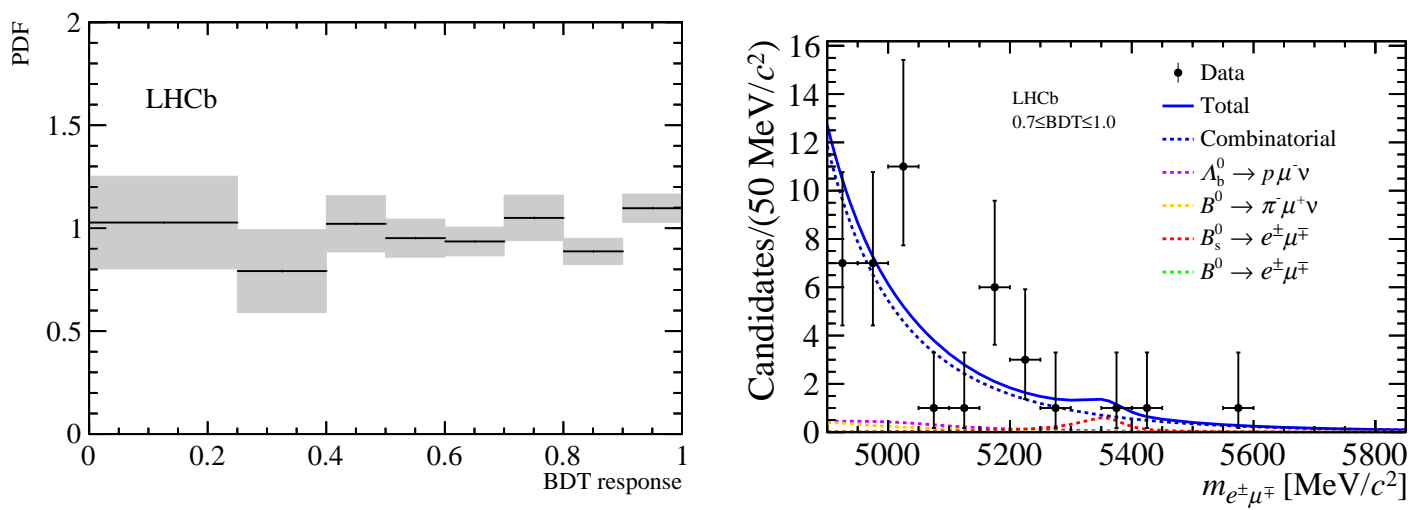

Figure 2: Fraction of $B^{0} \rightarrow K^{ \pm} \pi^{\mp}$ decays as a function of the classifier output. (left) Invariant mass distribution of the $e \mu$ system for $B_{(s)}^{0} \rightarrow e^{ \pm} \mu^{\mp}$ candidates integrated over all categories considered. (right)

\section{3. $\tau^{ \pm} \rightarrow \mu^{ \pm} \mu^{\mp} \mu^{ \pm}$}

The $\tau^{ \pm} \rightarrow \mu^{ \pm} \mu^{\mp} \mu^{ \pm}$decay was analysed at LHCb using $3 \mathrm{fb}^{-1}$ of data collected in Run I [7]. Events are studied in a binned three dimensional space where the dimensions are: the mass of the $\tau$ candidate and the output of two multivariate classifiers: one aimed at rejecting combinatorial background and based on topological information such as vertex quality and displacement and the second aimed at rejecting mis-identified decays and based on particle identification information. The $D_{s}^{+} \rightarrow\left(\phi \rightarrow \mu^{ \pm} \mu^{\mp}\right) \pi^{+}$decay is used for normalisation and its invariant mass is shown in Fig. 3 together with the invariant mass of the $\tau$ candidates. No signal excess is observed and a limit is set at $\mathcal{B}\left(\tau^{ \pm} \rightarrow \mu^{ \pm} \mu^{\mp} \mu^{ \pm}\right)<4 \times 10^{-8}$ at $90 \%$ confidence level, which is competitive with the best limit set by Belle [8]. Previously, LHCb had set limits also on lepton number and baryon number violating decays of $\tau$ [9].

\section{Conclusions}

The observation of LFV would be a clear sign of new physics and searching for these decays 

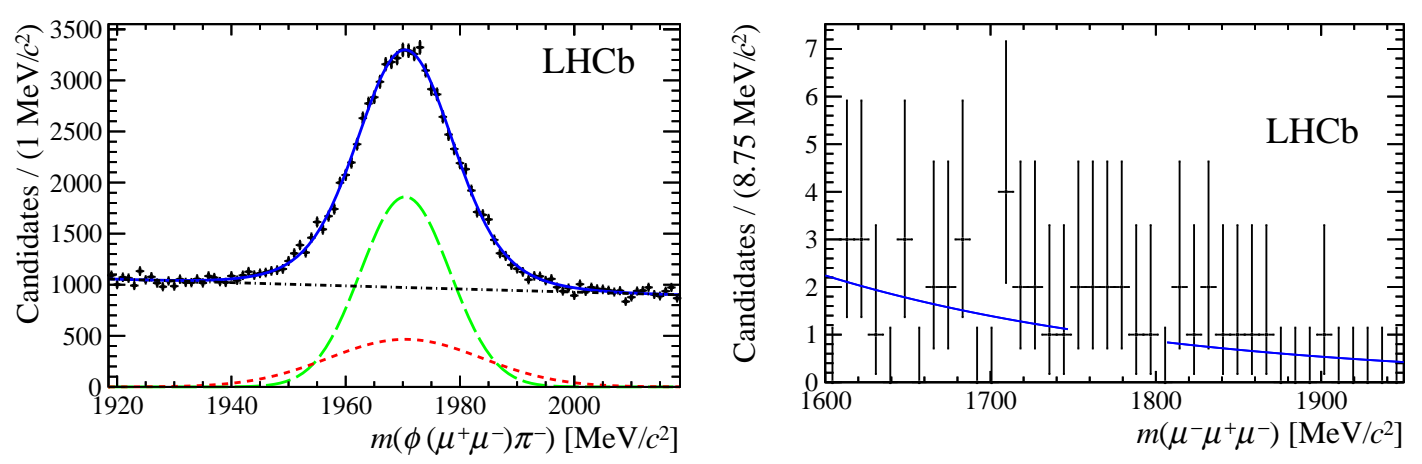

Figure 3: Invariant mass distribution for $D_{s}^{+} \rightarrow(\phi \rightarrow \mu \mu) \pi^{+}$(left) and $\tau^{ \pm} \rightarrow \mu^{ \pm} \mu^{\mp} \mu^{ \pm}$(right) candidates.

is particularly interesting because in many models they can be linked to the anomalies observed in LFU tests. LHCb searched for a variety of such decays: no signal excess is observed and therefore limits are set, which in several cases are the best to date. As in many channels the level of beyondSM predictions is within reach, $\mathrm{LHCb}$ will continue to analyse these decays including the Run II dataset and searching for new channels including semileptonic decays such as $B^{+} \rightarrow K e^{ \pm} \mu^{\mp}$ and $\Lambda_{b}^{0} \rightarrow \Lambda e^{ \pm} \mu^{\mp}$ and decays including $\tau$ leptons such as $B^{0} \rightarrow \tau^{ \pm} \mu^{\mp}$ and $B \rightarrow K^{(* 0)} \tau^{ \pm} \mu^{\mp}$.

\section{References}

[1] LHCb Collaboration, R. Aaij et al., Test of lepton universality using $B^{+} \rightarrow K^{+} \ell^{+} \ell^{-}$decays, Phys. Rev. Lett. 113 (2014) 151601, [arXiv: 1406 . 6482].

[2] LHCb Collaboration, R. Aaij et al., Test of lepton universality with $B^{0} \rightarrow K^{* 0} \ell^{+} \ell^{-}$decays, arXiv:1705.05802.

[3] LHCb Collaboration, R. Aaij et al., Search for the lepton-flavour violating decay $D^{0} \rightarrow e^{ \pm} \mu^{\mp}$, Phys. Lett. B754 (2016) 167-175, [arXiv: 1512 . 00322 ].

[4] G. Hiller, D. Loose, and K. Schönwald, Leptoquark Flavor Patterns \& B Decay Anomalies, JHEP 12 (2016) 027, [arXiv: 1609.08895].

[5] G. Burdman, E. Golowich, J. L. Hewett, and S. Pakvasa, Rare charm decays in the standard model and beyond, Phys. Rev. D66 (2002) 014009, [hep-ph/ 0112235$].$

[6] LHCb Collaboration, R. Aaij et al., Search for the lepton-flavour violating decays $B_{(s)}^{0} \rightarrow e^{ \pm} \mu^{\mp}$, JHEP 03 (2018) 078, [arXiv: 1710.04111$].$

[7] LHCb Collaboration, R. Aaij et al., Search for the lepton flavour violating decay $\tau \rightarrow \mu \mu \mu$, JHEP 02 (2015) 121, [arXiv:1409.8548].

[8] K. Hayasaka et al., Search for Lepton Flavor Violating Tau Decays into Three Leptons with 719 Million Produced Tau+Tau-Pairs, Phys. Lett. B687 (2010) 139-143, [arXiv:1001.3221].

[9] LHCb Collaboration, R. Aaij et al., Searches for violation of lepton flavour and baryon number in tau lepton decays at LHCb, Phys. Lett. B724 (2013) 36-45, [arXiv: 1304 . 4518].

[10] LHCb Collaboration, R. Aaij et al., Search for Majorana neutrinos in $B^{-} \rightarrow \pi^{+} \mu^{-} \mu^{-}$decays, Phys. Rev. Lett. 112 (2014), no. 13 131802, [arXiv:1401.5361].

[11] LHCb Collaboration, R. Aaij et al., Search for $D+(s)$ to $p i+m u+m u-$ and $D+(s)$ to $p i-m u+m u+$ decays, Phys. Lett. B724 (2013) 203-212, [arXiv:1304.6365]. 